\title{
Closed-Form Solution of Large Deflected Cantilever Beam against Follower Loading Using Complex Analysis
}

\author{
Ibrahim M. Abu-Alshaikh ${ }^{1}$ \\ ${ }^{1}$ Department of Mechanical Engineering, University of Jordan, Jordan \\ Correspondence: Department of Mechanical Engineering, University of Jordan, Amman-11942, Jordan. E-mail: \\ i.abualshaikh@ju.edu.jo
}

Received: October 1, 2017

Accepted: October 18, $2017 \quad$ Online Published: November 21, 2017

doi:10.5539/mas.v11n12p12

URL: https://doi.org/10.5539/mas.v11n12p12

\begin{abstract}
The literature reveals that the non-conservative deflection of an elastic cantilever beam caused by applying follower tip loading was investigated and solved by various numerical methods like: Runge Kutta, iterative shooting, finite element, finite difference, direct iterative and non-iterative numerical methods. This is due to the fact that the Euler-Bernoulli nonlinear differential equation governing the problem contains the "slope at the free end", this slope however needs special numerical treatment. On the other hand, some of these methods fail to find numerical solutions for extremely large loading conditions. Hence, this paper is aimed to obtain a closed-form solution for solving the large deflection of a cantilever beam opposed to a concentrated point follower load at its free end. This closed-form solution when compared with other conventional numerical approaches is characterized by simplicity, stability and straightforwardness in getting the beam deflection and slopes even for extremely large loading conditions. The closed-form solution is obtained by applying complex analysis along with elliptic-integral approach. Very good results were obtained when the elastica of the beam compared with that of various numerical methods which are used in analyzing similar problem.
\end{abstract}

Keywords: Large elastica deflection, Tip-follower loading, closed-form solution

\section{Introduction}

Many engineering fields use cantilever beams (CBs) in their applications. These attractive applications are based on the elastic behavior of the beam in its large deflection (LD) state. These applications are used by mechanics, mechatronics, civil and military industries as in ship and plane building, athletes in pole-vault sport, heavy bridges, roofed-structures, compliant mechanisms, cranes and highly-flexible manipulators.

Many researchers investigated the LD of CB loaded by a follower load (FL). The large deflected behavior of a CB subjected to a tip point load is numerically analyzed by applying the static and dynamic stability-criteria to both uniform and non-uniform cross-section. These numerical approaches used the iterative shooting with Runge-Kutta (RK) method or other techniques introduced by Rao B.N. and Rao G.V., 1987-1988. Different boundary conditions of non-rotational over-hanged beam with a middle support accompanied with FL using Newton's method have been further investigated by Wang et al., 1998. Assuming constant inclination to the deformed axis of the CB loaded by tip force, the LD is studied by utilizing a high-order RK numerical method, Mutyalarao et al., 2010. A uniform curvature curved prismatic CB with a follower end load is also investigated by applying an iterative shooting method to analyze its LD behavior, Nallathambi et al., 2010. In the three papers of Shvarsman, 2007, 2009 and 2013, a direct numerical method is applied to study the "elastica" of a straight non-uniform CB under a tip-concentrated load. In these papers, the load is always assumed following the beam axis with constant inclination angle. Without iterations, the non-uniform cross-section flexible elastic CBs subjected to both intermediate and end-FL are numerically analyzed by Shvarsman, 2009. Shvarsman, 2013 assessed the validity of the non-iterative direct method for considering the static analysis of the elastic curved $\mathrm{CB}$ against a tip point follower force (FF).

The literature reveals that the non-conservative deflection of elastic beams caused by applying follower tip loading was usually investigated and solved by various conventional numerical methods like: RK, iterative shooting, finite element, finite difference, or the direct iterative and non-iterative numerical methods. The need for the prediction of the slope angles at the free end of a CB for various end-loads directed many authors to solve the problem numerically, Mutyalarao et al., 2010, Nallathambi et al., 2010 and Shvarsman, 2007, 2009 and 2013. 
In this paper, a novel attempt is created to present an exact closed form solution for the "elastica" of a LD of CB under a concentrated follower end force for different force inclination angles. The most recent paper by Alkhaldi, et al., proposed a closed-form solution of the LD of spring-hinged beams subjected to non-conservative loading of different inclinations and tip-end concentrated couple. In this work, only the elliptic integral approach without introducing complex analysis is applied for solving the problem. According to the author's best knowledge, it is noticed that there is no available closed-form solution in literature for such a problem which combines the complex analysis together with the elliptic integral approach. Obtaining a closed-form solution with this mathematical technique of complex analysis and comparing its results with the other existing numerical solutions is the main goal of this paper.

\section{Problem Formulation}

Consider a uniform slender elastic inextensible $\mathrm{CB}$ of length $L$ and of a constant flexural rigidity $E I$. Suppose that the beam is subjected to a point follower right-end load $P$ which is inclined by an angle $\alpha$ from the beam axis. The load and the slope angle along the neutral axis of the deflected beam are represented by Cartesian coordinates $(x, y)$ and arc length $s$, respectively, Fig.1. Hence, the load is non-conservative; the inclination angle $\alpha$ of the follower tip load with respect to the deformed neutral axis of the beam remains constant after deformation. Under the assumption that the beam is of negligible weight; the equilibrium equations in $x$ and $y$-directions show that the force components are independent the arc length $s$. Let the angle $\phi(s)$ denotes the slope angle of elastica with respect to the positive $x$-direction, and $d s$ represents the infinitesimal arc-length along the beam. Thus, balancing the shear force acting on the beam leads to the relation, Shvartsman, 2007.

$$
\frac{\mathrm{d}^{2} \phi(s)}{\mathrm{d} s^{2}}=-\beta^{2} \sin (\phi(s)+\alpha-\phi(0)) \text { where } \beta^{2}=\frac{P}{E I}
$$

Equation (1) is a second order nonlinear differential equation that describes the behavior of the CB where $\phi(0)$ is the slope of the beam at the free end $(s=0)$. The prediction of the corresponding values of $\phi(0)$ for any right-end load $P$ is difficult. However, this difficulty directed researchers to solve the deflection problem by various numerical techniques, Mutyalarao et al., 2010, Nallathambi et al., 2010 and Shvarsman, 2007, 2009 and 2013. The other difficulty comes up from the existence of the nonlinearity term in the right hand side of Eq. (1). Assuming that the left-hand side of the beam is fixed and the right-hand side is free with concentrated FF, the boundary conditions at these supports are mathematically governed by the two relations

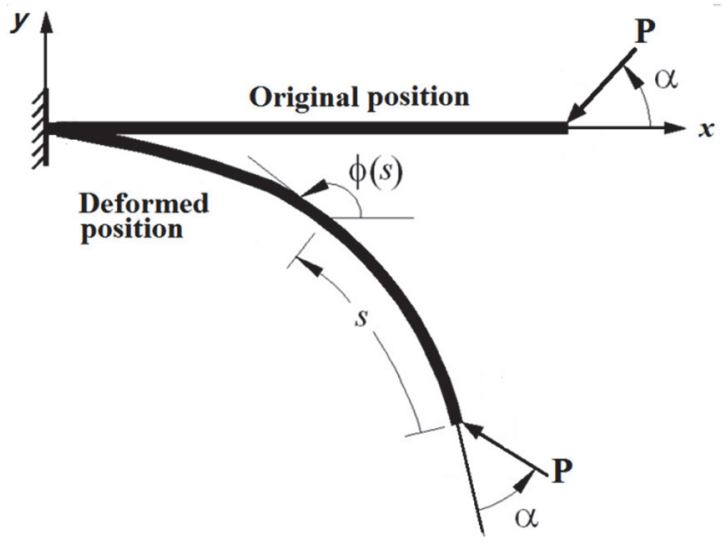

Figure 1. a CB subjected to end-concentrated FL inclined by an angle $\alpha$.

$$
\left.\frac{\mathrm{d} \phi(s)}{\mathrm{d} s}\right|_{s=0}=0, \phi(L)=0
$$

Once the nonlinear differential equation in Eq. (1) is solved for the slope angle $\phi(s)$, the $(x, y)$ coordinates of the beam-elastica are readily determined by integrating the following relations

$$
\frac{\mathrm{d} y}{\mathrm{~d} s}=\sin (\phi(s)), \frac{\mathrm{d} x}{\mathrm{~d} s}=\cos (\phi(s))
$$


By introducing angle transformation

$$
z(s)=\phi(s)+\alpha-\phi(0)
$$

where $z(0)=\alpha$. Thus, the beam Euler-Bernoulli equation, Eq. (1), using this transformation becomes

$$
\frac{\mathrm{d}^{2} z(s)}{\mathrm{d} s^{2}}=-\beta^{2} \sin (z(s))
$$

Multiplying both sides of Eq. (5) by the beam curvature $\mathrm{d} z(s) / \mathrm{d} s$ it becomes

$$
\frac{\mathrm{d}^{2} z(s)}{\mathrm{d} s^{2}} \frac{\mathrm{d} z(s)}{\mathrm{d} s}+\beta^{2} \sin (z(s)) \frac{\mathrm{d} z(s)}{\mathrm{d} s}=0
$$

Collecting the terms of Eq. (6) to be an argument for the first derivative $d / d s$, as

$$
\frac{\mathrm{d}}{\mathrm{d} s}\left[\frac{1}{2}\left(\frac{\mathrm{d} z(s)}{\mathrm{d} s}\right)^{2}-\beta^{2} \cos (z(s))\right]=0
$$

Equation (7) emphasizes the mechanical energy conservation principle of the beam and it can be integrated immediately by replacing the term inside the square brackets by an arbitrary constant $C$, as

$$
\left(\frac{\mathrm{d} z(s)}{\mathrm{d} s}\right)^{2}=\left[2 C+2 \beta^{2} \cos (z(s))\right]
$$

Here $C$ is a constant of integration that should satisfy the associated boundary conditions of the beam. Substituting the relations; $\cos (\mathrm{z}(\mathrm{s}))=1-2 \sin ^{2}(z(s) / 2)$ and $\sin (\mathrm{z}(\mathrm{s}) / 2)=\left[\mathrm{e}^{i z(s) / 2}-\mathrm{e}^{-i z(s) / 2}\right] / 2 i$ into Eq. (8), becomes

$$
\frac{\mathrm{d} z(s)}{\mathrm{d} s}=2 \beta \sqrt{\zeta^{2}-\left[\frac{\mathrm{e}^{i z(s) / 2}-\mathrm{e}^{-i z(s) / 2}}{2 i}\right]^{2}}
$$

where $i$ is the complex number defined by $i=\sqrt{-1}$. Applying the boundary condition at the free-end in cooperation with Eq. (4) leads that the constant $\zeta$ is

$$
\zeta^{2}=\frac{C+\beta^{2}}{2 \beta^{2}}=\sin ^{2}\left(\frac{\alpha}{2}\right)
$$

Hence, using Eqs. (2), (4) and (9), Eq. (10) can be derived by applying the boundary condition at the free end. Equation (9) corresponding to the beam curvature which can be examined to obtain the possible ranges of the slope angle at the free end. Since the curvature is definitely a real number, the sign under the square root in Eq.(9) should be definitely positive or zero along the beam. However, to satisfy this, the following mathematical condition must be met for each value of the arc length $s$

$$
\zeta^{2}=\sin ^{2}\left(\frac{\alpha}{2}\right) \geq \sin ^{2}\left(\frac{z(s)}{2}\right)
$$

Now, replacing the exponential terms in Eq. (9) by $\chi$, where

$$
\chi=\mathrm{e}^{i z(s) / 2}
$$

and by differentiating Eq. (12) with respect to $s$, it is possible to get the value of $(\mathrm{d} \chi / \mathrm{d} s)$ as 


$$
\left(\frac{\mathrm{d} \chi}{\mathrm{d} s}\right)=\frac{i}{2} \chi\left(\frac{\mathrm{d} z(s)}{\mathrm{d} s}\right)
$$

In view of Eq. (12), substituting Eq. (9) into (13) and rearranging the terms, Eq. (13) can be rewritten as

$$
\mathrm{d} s=\frac{\mathrm{d} \chi}{i \chi \beta_{1} \sqrt{\zeta^{2}+\frac{1}{4}\left[\chi-\frac{1}{\chi}\right]^{2}}}
$$

which is classified as a separable differential equation that can be solved by direct integration. In view of Eqs. (4) and (12), Eq. (14) can be solved for $\phi(s)$ as

$$
\phi(s)=-\alpha+\phi(0)+2 i \ln (A)-2 i \ln \left(s n\left[\frac{i A \beta}{2} s+F\left(A e^{i \alpha / 2} ; \overline{A^{2}}\right) ; \overline{A^{2}}\right]\right)
$$

where the bars over $A^{2}$ stands for the complex conjugate and the constant $A$ is defined by

$$
A=\sqrt{1-2 \zeta^{2}+\sqrt{4 \zeta^{2}\left(\zeta^{2}-1\right)}}
$$

Furthermore, Eq. (15) is written in terms of incomplete elliptic integral of the first kind $F(. ;$.) and in terms of Jacobi elliptic function $s n$ [. ; .]. Now, introducing the condition $\phi(\mathrm{L})=0$ at the fixed end, Eq. (15) can be solved exactly for $\phi(0)$ as

$$
\phi(0)=\alpha-2 i \ln (A)+2 i \ln \left(\operatorname{sn}\left[\frac{i A \beta}{2} L+F\left(A e^{i \alpha / 2} ; \overline{A^{2}}\right) ; \overline{A^{2}}\right]\right)
$$

At this end, one can find the Cartesian coordinates in both directions $(x, y)$ of the beam-elastica by substituting Eqs. (4), (8) and (10) into Eq. (3), where $\mathrm{d} z=\mathrm{d} \phi$ as

$$
\begin{aligned}
& x=\int_{\phi}^{0} \frac{\cos (\phi) \mathrm{d} \phi}{\sqrt{4 \beta^{2} \zeta^{2}-2 \beta^{2}+2 \beta^{2} \cos (\phi-\Omega)}}=M(0)-M(\phi(\mathrm{s})) \\
& y=\int_{\phi}^{0} \frac{\sin (\phi) \mathrm{d} \phi}{\sqrt{4 \beta^{2} \zeta^{2}-2 \beta^{2}+2 \beta^{2} \cos (\phi-\Omega)}}=N(0)-N(\phi(\mathrm{s}))
\end{aligned}
$$

where $\Omega=\phi(0)-\alpha$ is the difference between the slope angle at the free end and the inclination of the load angle. The two equations presented in (18) can be integrated numerically or analytically to find the results of the deflection of the elastica of the beam at any value of $s$, the analytical integration leads that

$$
\begin{aligned}
& M(\phi)=S_{5} B+S_{1} G\left[2 \zeta^{2} E\left(\mathrm{~S}_{4} ; S_{3}\right)-F\left(\mathrm{~S}_{4} ; S_{3}\right)\right] \\
& \mathrm{N}(\phi)=-S_{1} B+S_{5} G\left[2 \zeta^{2} E\left(\mathrm{~S}_{4} ; S_{3}\right)-F\left(\mathrm{~S}_{4} ; S_{3}\right)\right]
\end{aligned}
$$

Where $F(. ;$.$) is the incomplete elliptic integral of the first kind, and E(. ;$.$) is the incomplete elliptic integral$ function of the second kind, and 


$$
\begin{aligned}
& G=\frac{\mathrm{S}_{4}\left(1-\zeta^{2}\right)}{S_{6} \beta^{2} \sqrt{2 \beta^{2}\left(S_{2}+2 \zeta^{2}-1\right)}} \sqrt{\frac{1-S_{2}}{\zeta^{2}}} \sqrt{\frac{1+S_{2}}{1-\zeta^{2}}}, B=\frac{1}{2 \beta^{4}} \sqrt{2 \beta^{2}\left(S_{2}+2 \zeta^{2}-1\right)} \\
& S_{1}=2 \beta^{2} \cos (\Omega), S_{2}=\sin (\phi+\arcsin (\cos \Omega)), S_{3}=\sqrt{\frac{\zeta^{2}-1}{\zeta^{2}}} \\
& S_{4}=\sqrt{\frac{2 \zeta^{2}+S_{2}-1}{2 \zeta^{2}-2}}, S_{5}=2 \beta^{2} \sin (\Omega), S_{6}=\cos (\phi+\arcsin (\cos \Omega))
\end{aligned}
$$

Going back to Eq. (17), that represents a unique mathematical relation between the slope angles of the beam at the free end $\phi(0)$ with the ratio of the load to the flexural rigidity of the beam $\beta$. However, in view of this relation, Eq. (17), no need to predict the values of $\phi(0)$ or use any numerical technique or any shooting method to solve for any FL inclined by an angle $\alpha$, therefor the prediction is totally avoided. Thus, Eq. (17) combined with Eq. (15) represents the exact solution for the slope angle of the elastica of the CB that is subjected to the concentrated FF inclined by an angle $\alpha$. Furthermore, Eqs. (18-19) can also be used to find the exact deflection of the elastica of the beam at any value of S. However if $\phi(s)$ is replaced by $\phi(0)$ in Eq. (18) the exact deflection at the free end can be found. In the next Section, some numerical examples and verifications will be discussed.

\section{Results and Discussion}

In some numerical computations, it is suitable to introduce the following non-dimensional quantities

$$
\bar{s}=\frac{s}{L}, \bar{\beta}=\beta L=\sqrt{\bar{P}}, \bar{P}=\frac{P L^{2}}{E I}
$$

The curve shown in Fig. 2 which is drawn using Eq. (17) shows how the load variation affects the beam tip-angle for the case when a perpendicular FL is applied for load-with $\alpha=\pi / 2$. From Fig. 2 it can be noticed that; for a specified load only one tip-angle can be observed and not more, on the other hand, for a specified tip-angle many different loads can be observed. However, many loads for a beam tip-angle show different deformed configurations of the beam, which implies uniqueness on the deflection solution of the nonlinear differential equations of the investigated CB. Furthermore, the results presented in Fig.2 agree with the numerical and graphical results presented in reference, Mutyalarao et al., 2010, Shvarsman, 2007, 2013. It can be further concluded from Fig. 2 that the range of the tip-angle of the $\mathrm{CB}$ does not exceed double of the value of the inclination angle of the load, this conclusion can be further observed from Fig. 3 which is drawn for four different values of $\alpha$.

Table1 presents some numerical results obtained from the closed form solution using complex analysis corresponds to some selected points from Fig. 2, the table shows how these results fit exactly the numerical results presented by other researchers, Mutyalarao et al., 2010, Shvarsman, 2007, 2013. The obtained results from Eq. (15) and (17) which are illustrated in Table 1 are accurate even for large loads which may not be handled by some other numerical techniques.

Another verification problem developed in this paper appears by substituting Eq. (17) into Eq. (15) which leads to the exact solution of the problem under consideration. Ashok et al., treated this case and presented the deflection shape and the tip position for a wide range of values for the applied tip load. Ashok et. al. show how much it is easy to convert a two- parameter shooting method to a one-parameter shooting method by switching the direction of the numerical integration from the fixed end to the free end of the beam, and therefore avoiding the difficulties appeared in the two-parameter by shooting schemes, Ashok et al. 2010. Furthermore, other researchers apply the minimum potentail energy approach and the variational principle for modeling the LD behavior of beams with different boundary conditions loaded with follower type loadings, Ashok et al. and Dado, AL-Sadder. For the beam and load parameters $L=1000 \mathrm{~mm}, I=1.667 \times 10^{4} \mathrm{~mm}^{4}, E=210 \mathrm{GPa}$, the beam-elastica curves shown in Figs. 4 and 5 are depicted directly using the closed-form formula derived in Eq. (15) for fourteen different values of the FF with inclinations $\alpha=\pi / 2$ and $\alpha=\pi / 3$, respectively. The curves of Fig. 4 that represent the deformed shape of the $\mathrm{CB}$ agree exactly with those curves obtained numerically by different techniques in, Ashok et al. and Dado, AL-Sadder. 
One more verification problem is carried out by using the non-dimensional quantities presented in Eqs. (21). Figures 6 and 7 show the deformed configurations of the beams having the specified tip-angle with different non-dimensional load parameters inclined by the angles $\alpha=\pi / 2$ and $\alpha=\pi / 3$, respectively. From these figures it can be noticed that the deformation pattern for a specified tip-angle $\phi(0)$ differs due to different values of the load parameters. The results shown in Fig. 6 fit only the first five significant figures of the numerical results presented by Mutyalarao et al. This discrepancy in the results is due to the fact that the formulation presented here is exact while it was numerical to a certain margin of accuracy in Mutyalarao et al.

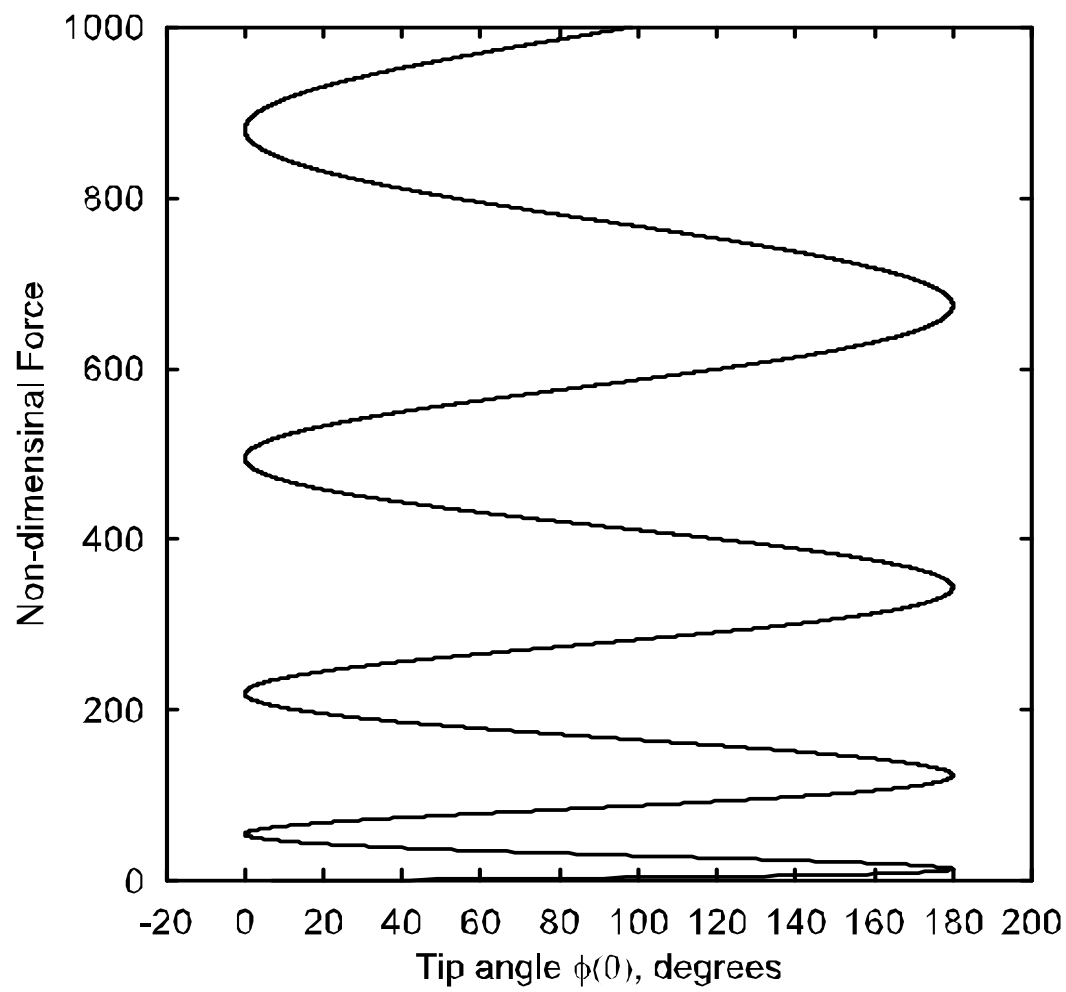

Figure 2. Variation of the tip-angle $\phi(0)$ of the $\mathrm{CB}$ with non-dimensional load $P$ or the load with $\alpha=\pi / 2$

Table 1. Comparison with literature fort $\alpha=\pi / 2$

\begin{tabular}{|c|c|c|c|c|}
\hline $\bar{P}$ & & Exact solution (Present) & Mutyalarao et al. 2010 & Shvartsman 2007 \\
\hline \multirow{3}{*}{30.938} & $\phi(0)$ & $90.002448689^{\circ}$ & $90^{\circ}$ & \\
\hline & $x(0) / \mathrm{L}$ & 0.4569544964 & 0.457054 & \\
\hline & $\mathrm{y}(0) / \mathrm{L}$ & -0.2542351094 & -0.254316 & \\
\hline \multirow{3}{*}{599.373} & $\phi(0)$ & $119.999363361^{\circ}$ & $120^{\circ}$ & \\
\hline & $x(0) / \mathrm{L}$ & 0.3755191709 & 0.366661 & \\
\hline & $\mathrm{y}(0) / \mathrm{L}$ & 0.2788732142 & 0.297055 & \\
\hline \multirow{3}{*}{1113.780} & $\phi(0)$ & $180^{\circ}$ & $180^{\circ}$ & \\
\hline & $x(0) / \mathrm{L}$ & $-4.6022 \times 10^{-8}$ & 0.000824 & \\
\hline & $\mathrm{y}(0) / \mathrm{L}$ & 0.4569466021 & 0.460062 & \\
\hline \multirow{3}{*}{13.75} & $\phi(0)$ & $179.999999928^{\circ}$ & $180^{\circ}$ & $180^{\circ}$ \\
\hline & $x(0) / \mathrm{L}$ & 0.00001351337 & 0.000007 & 0.0000 \\
\hline & $\mathrm{y}(0) / \mathrm{L}$ & 0.4569527562 & 0.456957 & 0.4570 \\
\hline
\end{tabular}




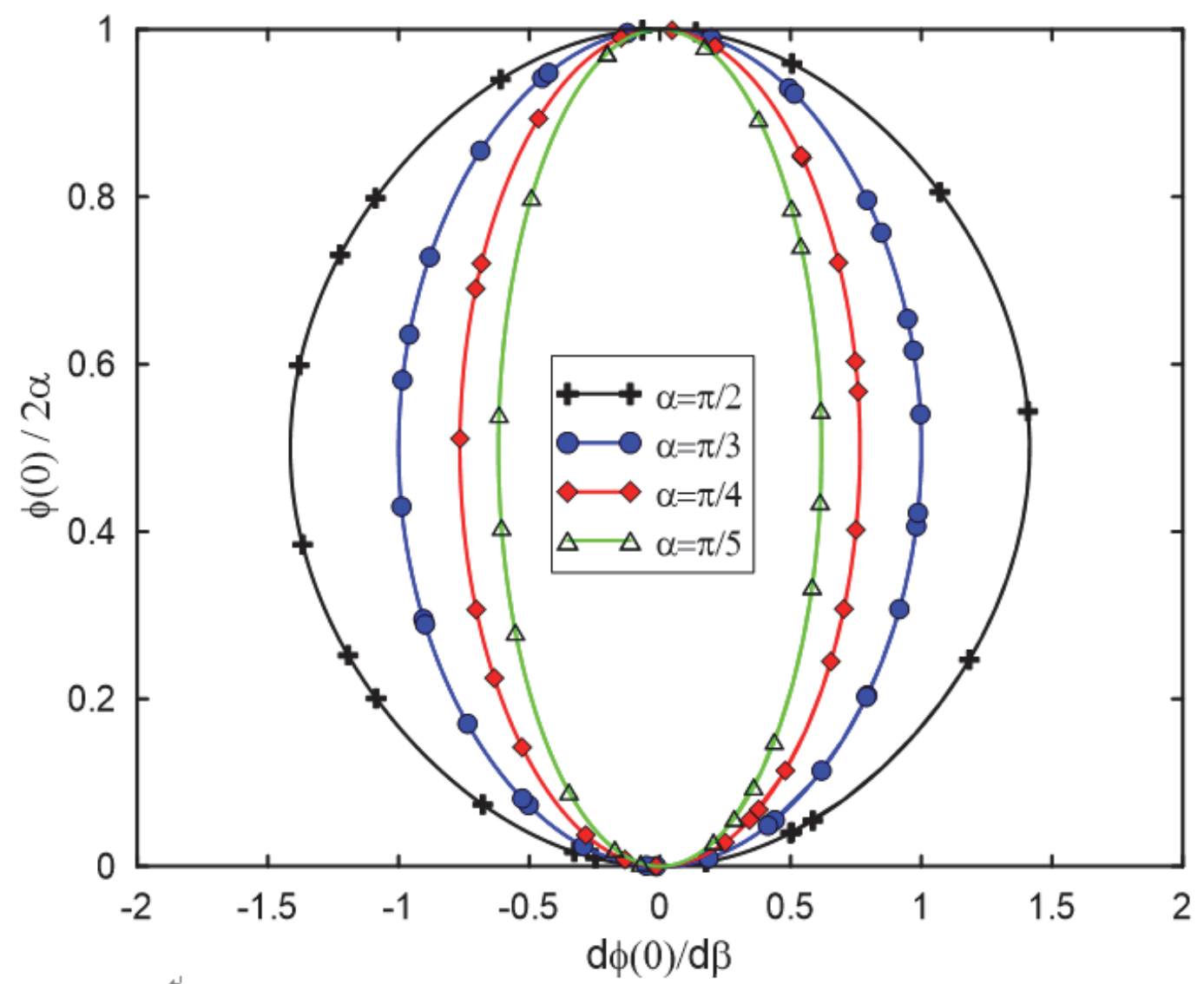

Figure 3. Variation of the $d \phi(0) / d \beta$ with the ratio $\phi(0) / 2 \alpha$ for various values of $\alpha$

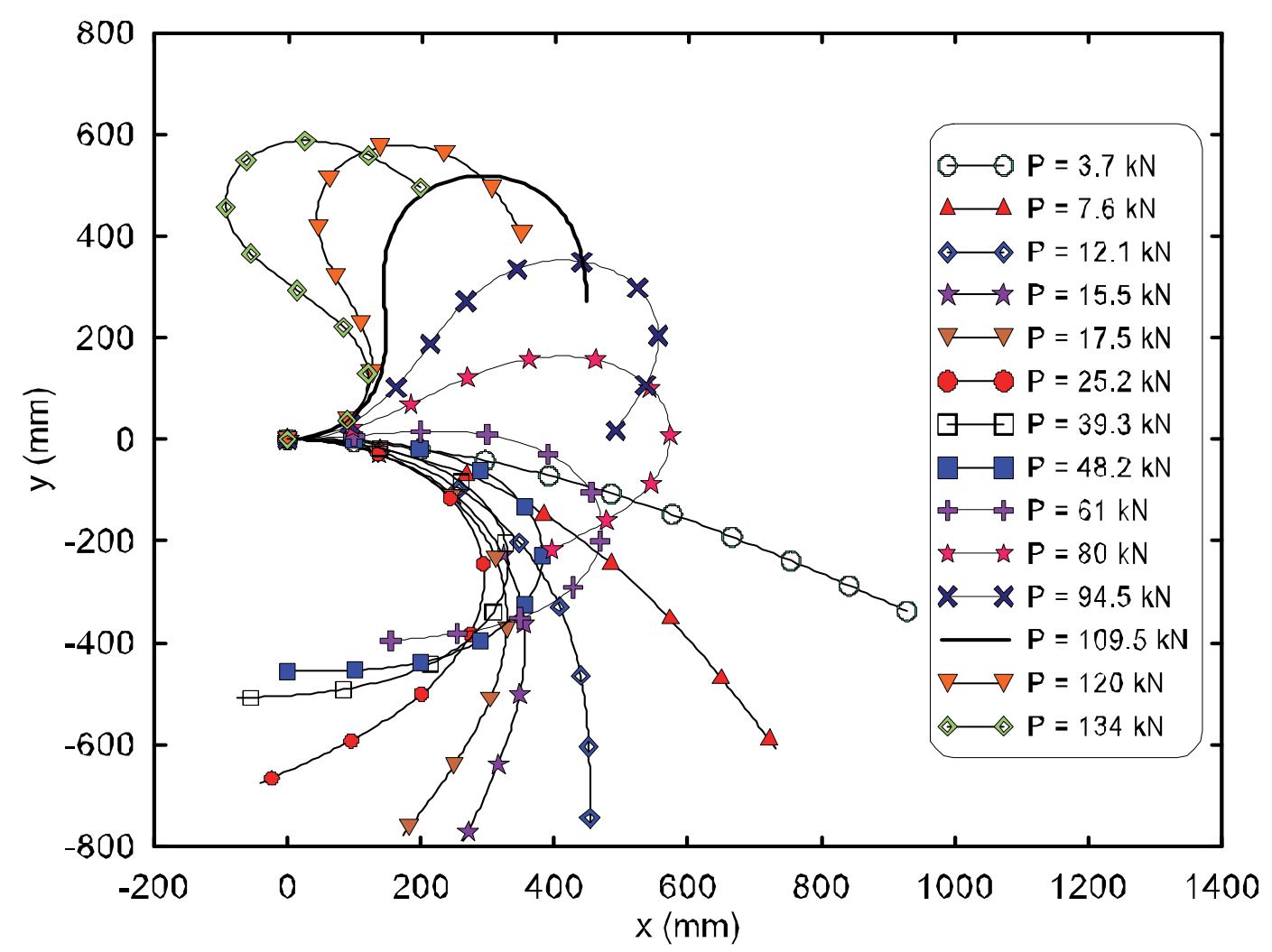

Figure 4. Deformed configurations of CB for some selected FFs with $\alpha=\pi / 2$ 


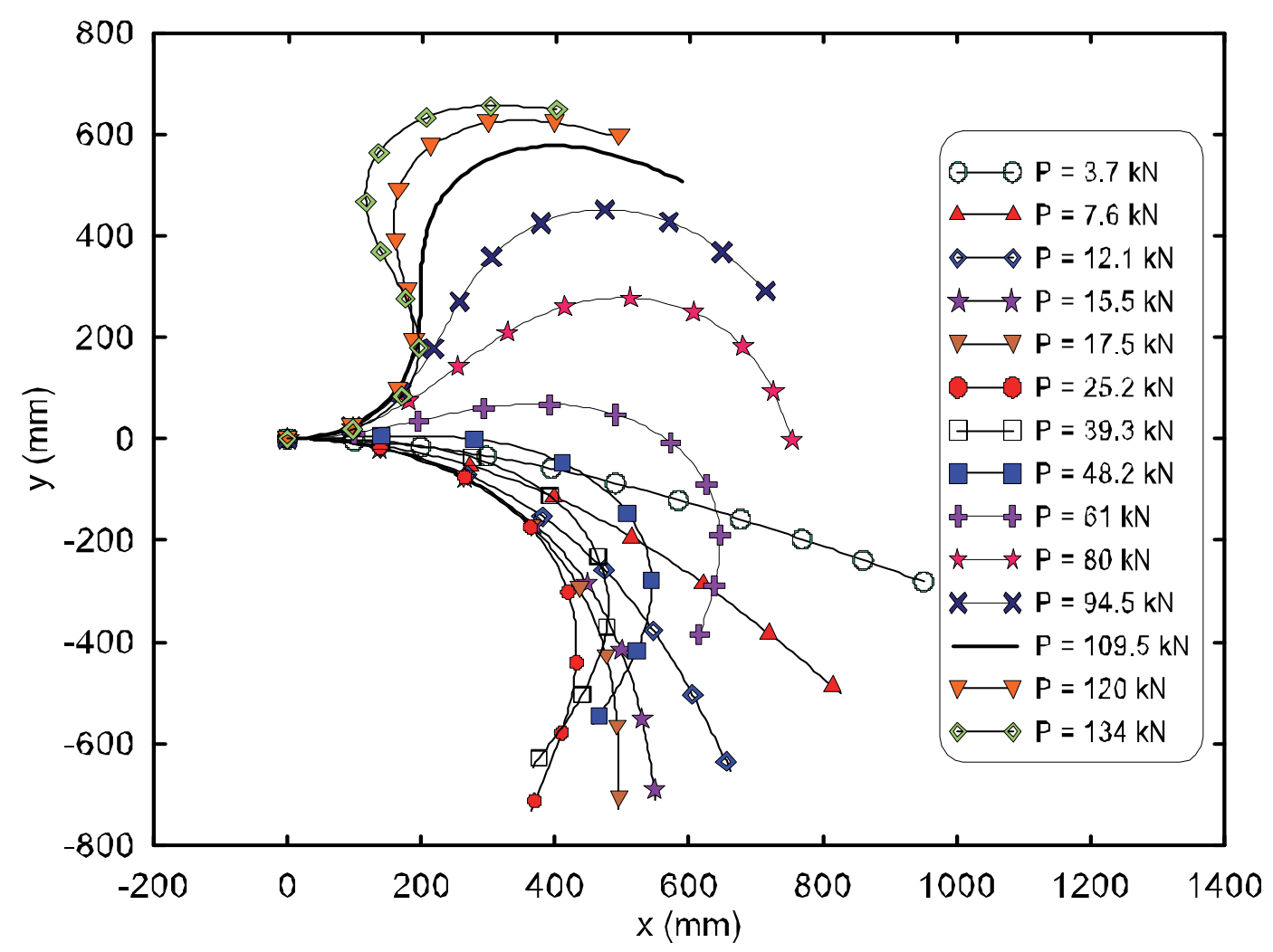

Figure 5. Deformed configurations of CB for some selected FFs with $\alpha=\pi / 3$

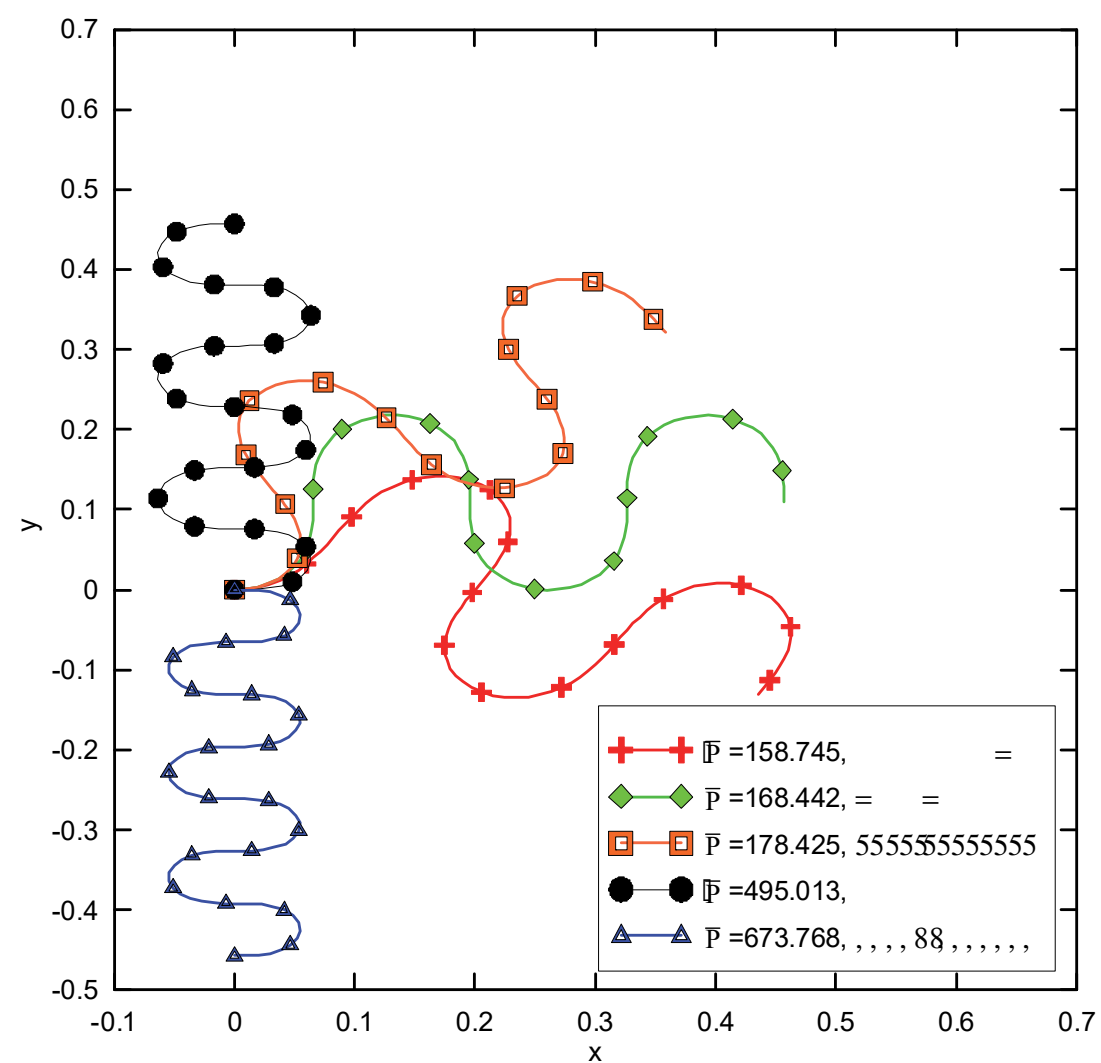

Figure 6. Deformed configurations of CB for some extreme non-dimensional forces $\bar{P}$ with $\alpha=\pi / 2$ 


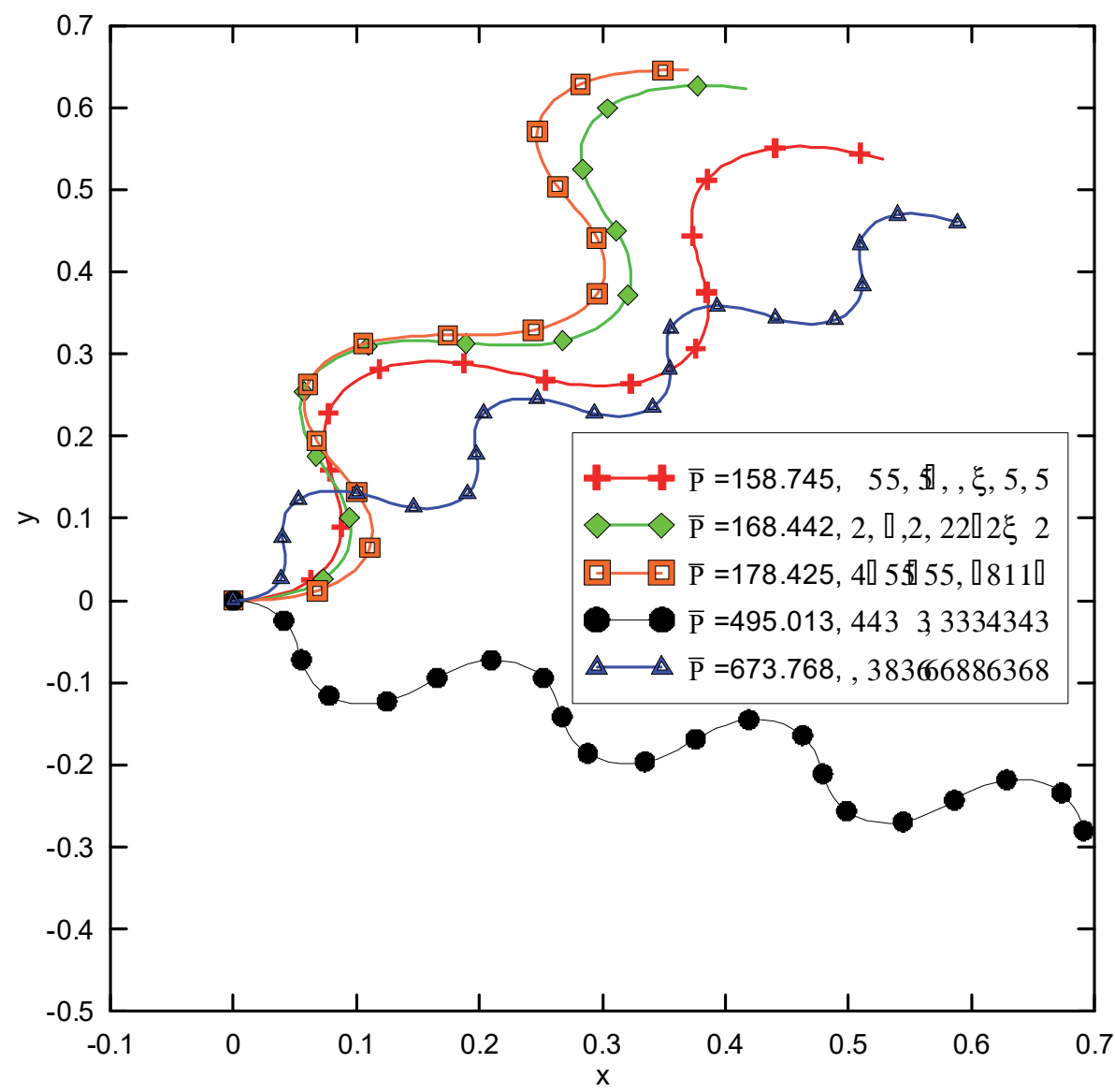

Figure 7. Deformed configurations of CB for some extreme non-dimensional forces $\bar{P}$ with $\alpha=\pi / 3$

\section{Conclusions}

The closed-form solutions of LD of CB subjected to a concentrated non-conservative inclined tip force are derived and verified by applying elliptic integral along with complex analysis. The exact solution for the tip-angle $\phi(0)$ is mathematically extracted while it is determined numerically by other researchers. The closed-form solution for any arbitrary slope angle $\phi(s)$ is characterized by its stability due to the mathematical fact that it contains exponential and complex functions. The technique used in this paper may also be applied to other beams with different boundary conditions and it may be further applied for multi-FLs distributed along the beam.

\section{References}

Alkhaldi H., Abu-Alshaikh I., Abu-Mallouh R., and Ghazal O., 2014, Closed-form solution of large deflection of a spring-hinged beam subjected to non-conservative force and tip end moment. European Journal of Mechanics-A/Solids, 47, 271-279. https://doi.org/10.1016/j.euromechsol.2014.02.019

Ashok, K. N., Lakshmana, C. R., \& Sivakumar, M. S. (2010). Large deflection of constant curvature cantilever beam under follower load, International Journal of Mechanical Sciences, 52, 440-445. https://doi.org/10.1016/j.ijmecsci.2009.11.004

Dado, M., \& AL-Sadder, S. (2005). A new technique for large deflection analysis of non-prismatic cantilever beams, Mechanics Research Communications, 2 32, https://doi.org/10.1016/j.mechrescom.2005.01.004.

Mutyalarao, M., Bharathi, D., \& Nageswara, R. B. (2010). Large deflections of a cantilever beam under an inclined end load, Applied Mathematics and Computations, 217, 3607-3613. https://doi.org/10.1016/j.amc.2010.09.021

Nallathambi, A. K., Rao, C. L., \& Srinivasan, S. M. (2010). Large deflection of constant curvature cantilever beam under follower load, International Journal of Mechanical Sciences, 52, 440-445. 
https://doi.org/10.1016/j.ijmecsci. 2009.11.004

Rao, B. N., \& Rao, G. V. (1987). Applicability of static and dynamic criterion for the stability of a cantilever column under a tip-concentrated sub-tangential follower force, Journal of Sound Vibration, 120, 197-200. https://doi.org/doi.org/10.1016/0022-460X(88)90345-8

Rao, B. N., \& Rao, G. V. (1988). Applicability of static or dynamic criterion for the stability of a non-uniform cantilever column subjected to a tip-concentrated sub-tangential follower force, Journal of Sound Vibration, 122, 188-191. https://doi.org/doi.org/10.1016/S0022-460X(88)80017-8

Shvartsman, B. S. (2007). Large deflections of a cantilever beam subjected to a follower force. Journal of Sound Vibration, 304, 969-973. https://doi.org/10.1016/j.jsv.2007.03.010

Shvartsman, B. S. (2009). Direct method for analysis of flexible cantilever beam subjected to two follower forces, International Journal of Non-Linear Mechanics, 44, 249-252. https://doi.org/10.1016/j.ijnonlinmec.2008.11. 004

Shvartsman, B. S. (2013). Analysis of large deflections of a curved cantilever subjected to a tip-concentrated follower force, International Journal of Non-Linear Mechanics, 50, 75-80. https://doi.org/doi.org/10.1016/j.ijnonlinmec.2012.10.015

Wang, C. M., Lam, K. Y., \& He, X. Q. (1998). Instability of variable arc-length elastica under follower force, Mechanics Research Communications, 25, 189-194. P1l S0093-6413(98)0002,1-X.

\section{Copyrights}

Copyright for this article is retained by the author(s), with first publication rights granted to the journal.

This is an open-access article distributed under the terms and conditions of the Creative Commons Attribution license (http://creativecommons.org/licenses/by/4.0/). 